\title{
Multicellular rosettes organize neuropil formation
}

\section{Authors}

Christopher A. Brittin ${ }^{1,{ }^{*}}$, Anthony Santella ${ }^{1,2,{ }^{*}}$, Kristopher Barnes ${ }^{1,3,{ }^{*}}$, Mark W. Moyle ${ }^{4}$, Li Fan ${ }^{1,+}$, Ryan Christensen $^{5}$, Irina Kolotuev ${ }^{6}$, William A. Mohler ${ }^{7,9}$, Hari Shroff ${ }^{5,9}$, Daniel A. Colón-Ramos ${ }^{4,9}$, Zhirong $\mathrm{Bao}^{1, q, \wedge}$

\section{Affiliations}

${ }^{1}$ Developmental Biology Program, Memorial Sloan Kettering Cancer Center, New York, NY 10065, USA

${ }^{2}$ Molecular Cytology Core, Memorial Sloan Kettering Cancer Center, New York, NY 10065, USA

${ }^{3}$ Graduate Program in Neuroscience, Weill Cornell Medicine, 1300 York Avenue, New York, NY 10065, USA

${ }^{4}$ Department of Neuroscience and Department of Cell Biology, Yale University School of Medicine, New Haven, CT 06536, USA

${ }^{5}$ Laboratory of High Resolution Optical Imaging, National Institute of Biomedical Imaging and Bioengineering, National Institutes of Health, Bethesda, MD 20892, USA

${ }^{6}$ EMF Facility, Universite de Lausanne, Batiment Biophore, Quartier Sorge, CH-1015 Lausanne, Switzerland

${ }^{7}$ Department of Genetics and Genome Sciences and Center for Cell Analysis and Modeling, University of Connecticut Health Center, Farmington, CT 06030, USA

+ Current address: Helen and Robert Appel Alzheimer's Disease Research Institute, Weill Cornell

Medicine, 1300 York Avenue, New York, NY 10065, USA

* Equal contribution

I Co-corresponding authors

$\wedge$ Correspondence to: baoz@mskcc.org 


\section{Summary}

2 Neuropils are compartments in the nervous system containing dense networks of neurites and

3 synapses which function as information processing centers. Neuropil formation requires

4 structural and functional organization at and across different scales, achieving single-axon

5 precision for circuits that carry out the core functions while simultaneously accommodating

6 variability among individuals $[1 ; 2 ; 3 ; 4]$. How these organizational features emerge over

7 development is poorly understood. The nerve ring is the primary neuropil in C. elegans, and its

8 structure is thoroughly mapped $[5 ; 6]$. We show that prior to axon outgrowth, nerve ring neurons

9 form a ring of multicellular rosettes with surrounding cells to organize the stratified nerve ring

10 structure $[7 ; 8]$. Axon bundles which correspond to future nerve ring strata grow from rosette

11 centers, travel along the ring on "bridge" cells that are simultaneously engaged in adjacent

12 rosettes, and assemble into a topographic scaffold of the nerve ring. SAX-3/Robo is required for

13 proper rosette formation and outgrowth from the center. Furthermore, axon contact sites that

14 form early in development are more conserved than the later ones, indicating a temporal

15 component in neuropil structural variability. Our results reveal an unexpected and critical role of

16 collective cell behaviors prior to innervation to pattern a complex neuropil and orchestrate its

17 formation across scales. 


\section{Main}

Neuropil development has largely been considered within the context of molecular guidance cues and adhesion-based affinity between neurites $[3 ; 9 ; 10]$. However, it is still challenging to

21 extrapolate from these fundamental mechanisms the complex organization of neuropils.

22 Concepts such as collective behaviors of neurites and the interactome [10; 11] are raised to

23 account for emergent structural features, which starts to align the question of neuropil formation

24 to that of complex tissue morphogenesis [12; 13] where collective cell behaviors have been

25 examined for how tissue morphology arises.

26 The complete connectome of $C$. elegans was mapped to single-axon and single-synapse

27 resolution over three decades ago [5]. Recent analyses of the data have revealed that the nerve

28 ring exhibits three general concepts of neuropil organization. (i) Structural stratification of circuits

29 at the meso-scale [2; 14]: the nerve ring shows spatial stratification of axons [7; 8] with each

30 strata exhibiting its own sensorimotor synaptic pathway [7]. (ii) Functional hierarchy of

31 information flow [2; 14]: nerve ring interneurons can be placed into a functional layered

32 hierarchy with prominent feedforward loops between layers [6; 7]. (iii) A conserved core circuit

33 embedded in an individual variable background [14; 15]: despite the long-held view of an

34 invariant connectome, the nerve ring shows considerable variability in axon positions - only 40\%

35 of axon contacts within the nerve ring are conserved between individuals [7].

36 As in other neuropils, how the $C$. elegans nerve ring acquires its structural features over

37 development is largely unknown. Several guidance molecules are secreted at different positions 
along the future nerve ring $[16 ; 17]$. A small group of neurons have been identified as pioneer

39 axons and interact with glia cells to guide the followers [17; 18], but it is unclear how the stratified

40 structure arises in this process. Here, we show that the formation of multicellular rosettes, a

41 collective cell behavior observed in diverse developmental contexts across species [19-23],

42 occurs among nerve ring neurons and surrounding tissues and plays a key role in organizing the

43 structure and developmental dynamics of nerve ring formation.

\section{Neurons form a ring of rosettes along the future nerve ring prior to axon growth}

45 To understand the dynamic cellular interactions that lead up to axon outgrowth and nerve ring

46 formation, we conducted 3D, time lapse imaging with a broadly expressed membrane marker to

47 examine cell shape and a ubiquitously expressed histone marker to trace and assign lineage

48 identity to every cell [24]. At approximately 350 min post fertilization (mpf), just prior to initial

49 axon outgrowth, we observed that the nerve ring neurons and their surrounding cells are

50 organized into 8 multicellular rosettes (Fig 1a) that are both spatiotemporally and

51 compositionally stereotyped as confirmed by both fluorescence imaging ( $n=3$, Fig 1b,c, Table S1,

52 Fig S1) and electron micrograph (EM) reconstruction ( $n=1$, Fig 1d). Rosettes form by contraction

53 of cell-cell contacts and are recognized by the convergence of multiple cells to a central foci [25].

54 Each rosette contains both neuronal and non-neural cells (Table S1) -- pharyngeal cells, muscle,

55 the glia-like GLRs, or excretory pore cells -- all of which have been postulated as scaffolds for the

56 C. elegans nervous system [26]. 
57 The 8 rosettes are ordered in a bilateral (left/right) symmetric circle around the pharynx that

58 corresponds to the path of nerve ring innervation (Fig 1a). At the anterior-dorsal end, the Dorsal

59 rosette sits on the dorsal midline. At the posterior-ventral end, the Excretory Pore rosette sits on

60 the ventral midline. Between these two rosettes are the bilaterally symmetric Distal Lateral

61 (DistL), Proximal Lateral (ProxL) and Sublateral (SubL) rosettes. While most rosettes (6/8) form

62 by simple multicellular convergence, the SubL Rosette, which contains 27 cells, is first formed as

63 three intermediate rosettes (anterior, medial and posterior precursors, Fig S1) that coalesce into

64 one rosette through contraction of cell-cell contacts.

65 Intermediate 'bridge cells' link subsequent rosettes into a single ring. Adjacent rosettes are

66 coupled by 1-3 intermediary cells that simultaneously engage both rosettes (Fig 1a,b,c), which

67 we term bridge cells (Table S1). From the Dorsal rosette to the SubL, the bridge cells are

68 predominantly papillary sensory neurons. Between the SubL and Pore rosettes, the bridge cells

69 include both interneurons and structural cells in the excretory pore.

70 In summary, our observations suggest a two-component --- rosettes and bridge cells -- structural

71 model that supports nerve ring formation. The rosettes pattern neurons along the path of the

72 nerve ring while bridge cells physically link rosettes along the path. Based on these observations

73 we made two hypotheses: (i) rosettes neurons support the structural stratification of the nerve

74 ring and (ii) bridge cells guide axons along the path of the nerve ring. 
Rosette axons form the topographical scaffold of the nerve ring

76 Cross examination of these rosettes and the nerve ring strata suggests that these rosettes

77 correspond to the topographic features of the nerve ring. Excluding the sensory axons which grow late [8] and axons with complex trajectories that cross multiple strata [7], there are 26 axons originating from the rosettes. We find a clear correspondence between the rosette cells and nerve ring strata (Fig 2a,b). The DistL and ProxL rosettes correspond to Strata D and B, respectively. The majority of the SubL axons corresponds to Stratum C, while the rest are in Stratum B -- in different analyses Stratum B and C may be merged into one stratum depending on the cutoff of clustering and differential weighting on axon contact [7; 8]. The Dorsal rosette, which will eventually split to form the anterior (RME and RMED) and posterior (ALA) boundaries of the nerve ring, includes cells from Stratum A and E. Based on this correspondence, we hypothesized that the axonal outgrowth from the rosettes contribute to the stratified structure

87 of the nerve ring.

To examine the outgrowth of the 26 neurons with live fluorescence imaging, we screened for and identified a set of cell-specific markers (Dorsal rosette: ceh-10, DistL: zag-1, ProxL: cnd-1, ProxL and SubL: lim-4, Figure 2c,d, S2a) [27], which label 21 of the 26 neurons. Axon outgrowth first appears from the SubL rosette at about $360 \mathrm{mpf}$ while outgrowths from the other rosettes enter the nerve ring shortly thereafter (Figure 2e). Bilateral axons meet at the dorsal midline to close the ring by $400 \mathrm{mpf}$. While the SubL axons (SIA, SIB and SMDD) serve as the pioneer for axons that enter the nerve ring at later stages and is indeed the first to grow among the rosette axons, our previous laser ablation that removed this group did not affect the outgrowth from the ProxL 
rosette [24]. These results suggest that rosette axons collectively form the initial nerve ring

97 without necessarily a pioneer-follower hierarchy among them.

98 Furthermore, we find that the relative placement of rosette axons within the nascent nerve ring

99 (Fig $\mathbf{2 d , f}$ ) corresponds to the same spatial order as their corresponding strata in the final nerve ring (Fig 2g). Two-color fluorescence imaging with pairwise combinations of the cell-specific markers (Fig 2c, S2a) were used to infer the relative positions between the labeled axons (Fig S2b)

102 in the nascent nerve ring. From anterior to posterior, the rosette axons are arranged as Doral 103 (RMED)/Stratum A, ProxL/Stratum B, SubL/Strata B and C, DistL/Stratum D, and Dorsal 104 (ALA)/Stratum E (Fig 2f). This suggests that rosette axons contribute to the initial stratification of 105 the nerve ring.

106 These structural and developmental analyses suggest a model where the rosette axons form 107 multiple pioneer bundles that in turn assemble into a scaffold of the nerve ring strata. Genetic 108 ablation of all purported pioneer neurons in the SubL and ProxL rosettes (excluding AVK) led to 109 profound defects in but did not completely abolish nerve ring formation [8]. Pioneering function 110 of the Dorsal and DistL axons remains to be tested.

111 Scaffold of pioneer axons is used to organize vertebrate brain development, where pioneer axons

112 originating from different regions of the brain lay the initial track for the major longitudinal and 113 circumferential tracts [4]. However, how these pioneer axons are globally organized is poorly 114 understood. Our results suggest rosettes as a novel mechanism to organize pioneer axons into a 115 scaffold of a neuropil. 


\section{Rosette centers coordinate polarized and collective axonal outgrowth}

117 We then asked how the ring of rosettes organizes the pioneer axons. Live fluorescence imaging

118 showed that axons grow out collectively from rosette centers (Fig 3a). Outgrowths from the SubL

119 rosette involve SIAD, SIBV and SMDD. Our previous studies ablating combinations of individual

120 neurons suggest that the observed extension contains outgrowth of each of these neurons [24].

121 In the DistL rosette, AVD and AVJ start outgrowth within 10 min of each other. However, not

122 every neuron in a rosette participates in this collective outgrowth, such as the sensory neurons

123 discussed above.

124 The rosette centers also show collective polarization. In particular, we found that SAX-3/Robo is

125 enriched at the center (Fig 3b,c, S3), but not PAR-6. SAX-3 is known to regulate neuronal

126 polarization and axon guidance [28], suggesting collective polarization.

127 Furthermore, we found that sax-3 is required for proper formation of the rosettes as well as the

128 collective outgrowth from the center. In 4 out of 14 embryos that are homozygous for the null

129 allele, $k y 123$, the posterior precursor rosette of the SubL rosette formed but failed to join the

130 anterior and medial precursor rosettes to form the complete SubL rosette. No outgrowths were

131 observed from the center of the isolated posterior precursor rosette either (Fig 3d). More subtle

132 phenotypes were also observed in terms of the stability of the rosettes: after joining a rosette a

133 cell may detach from the center and join again. In the ProxL rosette, which involves pharyngeal

134 cells, this is reflected in a less focused center that stays away from the boundary of the pharynx

135 (Fig 3d). 
136 Our results show a novel function of sax-3/Robo to regulate axon growth through rosette

137 formation, outside of its traditional role as a guidance receptor [28]. The incomplete penetrance

138 of $s a x-3$ phenotypes indicates that other pathways may be acting in parallel $[18 ; 22 ; 29]$.

139 Collective behaviors in axon outgrowth have been presented as a solution in axon targeting [3;

$14011]$, but it is unclear how these collective modules arise. Our results suggest rosette formation as

141 a novel mechanism that supports collective modules through collective polarization and

142 outgrowth. Intriguingly, the Drosophila ommatidia forms a rosette during its assembly [20] and

143 the axons subsequently undergo collective afferent sorting [3], although the connection between

144 the rosette and axon outgrowth has not been examined.

145 Axons travel along bridge cells to the next rosette

146 After the axon bundle emits from a rosette center, it appears to travel dorsally to the next rosette

147 center in the chain. Given that the adjacent rosettes are connected by bridge cells, we wondered

148 if these cells function to guide the axons between rosette centers.

149 EM images of nascent axons from the SubL rosette support this notion. Two bridge cells connect

150 the SubL rosette to the ProxL rosette, namely the IL2V neuron and the mother of the IL1V neuron

151 (denoted as IL1Vm for simplicity). These two cells are next to each other. On the left side of the

152 embryo, SIADL and SIBDL from the SubL rosette have started their outgrowth, and the

153 outgrowths are along IL1VLm and IL2V towards the ProxL rosette (Fig 3e). Similarly on the right

154 side of the embryo SIADR has started its outgrowth and grows along IL1VRm and IL2VR. 
155 To test if IL1Vm and IL2V are required to guide the axons, we used laser ablation to kill the 156 grandmother of these cells (Fig 3f) in 8 embryos. In 2 embryos, cell divisions near the targeted 157 cell were delayed, suggesting off-target effects (Fig S4). In 5 out of the 6 remaining cases, the 158 SubL neurons made a short outgrowth but did not extend further into the ProxL rosette as in the 159 wild type (Fig $\mathbf{3 f}, \mathbf{g}, p=5 \times 10$ ). The ablation did not affect the formation of the SubL rosette as 160 judged by the convergence of the labeled neurons, especially the elongated SIAV (Fig 3f). In 161 contrast, in control ablations to kill cells next to the grandmother of IL1Vm and IL2V, all SubL 162 outgrowths were wild type ( $n=6$, Fig $\mathbf{3 g}$ ). These results suggest that the phenotype is specific to 163 killing the grandmother of IL1Vm and IL2V. The ablation also removes BAG and SMDV. BAG is not 164 part of either rosette; SMDV is part of the ProxL rosette but on the opposite side of the SubL 165 rosette. Furthermore, it is unlikely that IL2V or IL1Vm can guide the SubL axons as classical 166 pioneer axons, because their axons are initiated at a much later stage [8].

167 Taken together, our imaging and ablation experiments suggest that bridge cells tile the path for 168 the pioneer axons and guide the axons from one rosette center to the next. This scheme would 169 allow the axon bundles from different rosettes to meet at rosette centers and sort their relative 170 positions to generate the topographic arrangement of the scaffold. That is, the ring of rosettes 171 also provides a sequence of decision points where the pioneer bundles sort into the neuropil 172 topography. 


\section{Rosettes reveal additional organizational schemes of the nerve ring}

174 Identification of the rosettes and the topographic scaffold allowed us to examine the pattern of

175 structural variability in the nerve ring. A recent analysis of EM data found that only $\sim 40 \%$ of axon

176 contact sites are conserved across animals [7]. Strikingly, 95\% (18/19) of the intra-rosette contact

177 sites are conserved, which is significantly higher than the overall $40 \%\left(p=1.8 \times 10^{-8}\right)$. In contrast,

178 only $31 \%(8 / 26)$ of contact sites between rosette axons are conserved (Fig 4 a). This contrast is

179 consistent with our model of scaffold assembly where pioneer bundles sort their relative

180 positions when they meet at rosette centers and suggests that coupling of axons is tight within

181 each pioneer bundle but relaxed between pioneer bundles. Interestingly, this pattern of

182 conserved intra- vs inter-rosette axon contact sites seems to extend to intra- and inter-stratum

183 axon contact sites (60\% vs 30\%, [7]).

184 We then asked if the order of entrance into the nerve ring may affect the variability of an axon's

185 position. To this end, we divided the nerve ring axons into three groups, namely early (rosette

186 neurons), late (tail and postembryonic neurons) and middle (others) axons. Given the difference

187 of intra- and inter-stratum conservation, we focused on intra-stratum contact sites. We found a

188 trend where the fraction of conserved contact sites decreases over developmental stages (Fig

189 4b): axons that enter later have less conserved contact sites than those that enter earlier. These

190 results suggest a developmental component in determining the individual variability of neuropil

191 structure. The pioneer scaffolds in the vertebrate brain are considered stereotypical [4]. Future

192 examination of axon positions at later developmental stages and finer resolution when the

193 technology is available would test if our observation represents a general principle. 
194 The rosettes also show a correlation with the functional organization of the connectome. Among

195 the pioneer bundles, 17 of the 26 axons are motoneurons (Fig $\mathbf{2} \mathbf{b}$, purple), which is a notably

196 higher fraction than that among head neurons (34/138). The 9 interneurons (Fig 2b, orange)

197 show close association with these motoneurons in the hierarchy of information flow from 198 sensory neurons to muscles [6, 30]. A recent study that examined the wiring diagram as a residual 199 network partitioned neurons into four layers in terms of the sensory-to-motor information flow 200 [7], Interestingly, all of the rosette neurons except for SIBD and AVJ are part of the layer for 201 sensorimotor convergence (Fig 4c). When interneurons are grouped into different layers by their 202 synaptic distance to motoneurons and the feedforward loops among them [6], all of the 9 203 interneurons except for ALA are in the first two layers of interneurons next to the motoneurons 204 (Fig 4c). Thus, contacts among the moto- and interneurons in the rosettes are likely part of the 205 core motor circuit. Given that these are pioneer neurons for the nerve ring and the inside-out 206 model of nerve ring growth where the sensory axons enter last [8], the order of nerve ring 207 entrance is in reverse order relative to the information flow (Fig 4d). As muscle movement is 208 required for embryo viability [31] and the 17 motoneurons in the rosettes collectively innervate 209 most of the muscles in the head and body [7], it is possible that neuropil formation is prioritized 210 for functional circuits to support development.

\section{Discussion}

212 Our results suggest that the ring of rosettes embodies key concepts in neuropil formation at 213 multiple levels of organization, including collective polarization of neurons, collective outgrowth 
214 of axons as bundles, path tiling for pioneer bundles, and assembly of pioneer bundles into a

215 topographic scaffold for the stratified structure of the neuropil. In particular, our results show

216 that a collective cell behavior prior to axon growth can function to organize a neuropil with

217 emergent topographic features. This finding provides a distinct perspective to the neurite-centric

218 view of the problem.

219 Because the complete cell lineage is known in C. elegans along with the complete connectome,

220 it has been a prime venue to examine the potential role of the cell lineage in shaping the

221 connectome. Early observations suggested that the invariant cell lineage positions lack obvious

222 correlation with the anatomy of the nervous system [32]. Rosette formation, which is inherently

223 based on local interaction of neighboring cells and apparently requires the right combination of

224 cell types given the invariant composition across embryos, can convert the spatial information in

225 cell position into the structure of the nerve ring through the steps discussed above.

226 Collective cell behaviors are increasingly recognized as an important paradigm for complex tissue

227 formation. Multicellular rosettes have been found in diverse contexts of neural morphogenesis

228 [20], such as the assembly of the Drosophila ommatidia, neuromast migration in zebrafish and 229 convergent extension of the mouse neural tube [21]. It will be interesting to see if rosettes also 230 play a role in axon and neuropil organization in organisms beyond C. elegans. 


\section{Methods}

232

\section{C. elegans strains and genetics}

233

C. elegans strains were raised at room temperature following the standard protocol. Strains and

234 genotypes used are listed in Table S3.

235 Live imaging of embryogenesis

236 Embryos were collected, mounted and imaged on a Zeiss AxioObserver Z1 inverted microscope

237 frame with Yokogawa CSU-X1 spinning disk, an Olympus UPLSAPO 60xs silicone oil immersion 238 objective and a pair of aligned EMCCD cameras (C9100-13) as previously described [22]. A stack 239 of images with 35 slices and 1 um between slices was collected every 1 to 3 minutes.

\section{Cell lineage tracing and laser cell ablation}

241 Real-time lineage tracing based on live imaging of embryogenesis and targeting of selected cells 242 were performed using the ShootingStar platform as previously described [24].

\section{Electron microscopy}

244 Embryos were prepared by High-Pressure Freezing followed by freeze substitution using $2 \%$ 245 osmium and 0.1\% Uranyl Acetate diluted in Acetone. After the dehydration cycles, samples were 246 gradually infiltrated in EPON 812 resin and flat embedded [33; 34]. An embryo at approximately $247360 \mathrm{mpf}$ was imaged with FEI HELIOS 650 FIB (Focused Ion Beam)-SEM microscope. The image 248 stack contains 944 sections at $30 \mathrm{~nm}$ apart with lateral resolution at $9.9 \mathrm{~nm}$. 
250 Fluorescence intensity measurements for SAX-3:GFP localization was done manually using FIJ.

251 Mean per-pixel intensity for a rosette center or a regular cell-cell junction was calculated in a

252 manually placed bounding rectangle. Background was estimated as mean per-pixel intensity of

253 all cells involved in a rosette or multicellular junctions and subtracted. Significance was calculated

254 between the two groups via 2-tailed T-test of equal variance.

2553 3 reconstruction from EM images were done manually using TrakEM2 [35]. Cell identities are 256 recognized by landmark cells with distinct shapes and relative cell positions that are invariant

257 across embryos based on live imaging of embryos with fluorescently labeled histones [27].

\section{Axon contacts and classification}

259 Strata in the nerve ring, association of axons with these strata, quantification of contact areas

260 between axons in an L4 and an adult nerve ring, as well as classification of these contacts in terms

261 of conservation were obtained from [7]. We defined an axon contact as conserved if its degree

262 of reproducibilty (degree) is 4 (Table S2), i.e. the axon contact is found on both the left and right

263 size of the nerve ring for both the L4 and adult EM reconstruction [7]. Then the 'fraction of axon

264 contacts conserved' is defined as the number of degree 4 contacts divided by the total number

265 of axon contacts (Fig $\mathbf{4 a , b}$ ). Intra-rosette contacts are defined as contacts between axons in the

266 same rosette (Table S1, intrarosette contact $=1$ ). Inter-rosette contacts are defined as contacts

267 between axons in different rosettes (intrarosette contact $=0$ ). The standard error is estimated 
268 by assuming that contacts are bionially distributed (i.e. either contacts are degree 4 or not degree 269 4).

270 Similar calculations were performed for early, middle and late axons (Fig 4b). Early axons are

271 defined to be the rosette axons. Late axons are axons from tail and postembryonic cells [26].

272 Middle axons are axons that are neither early nor late. Contacts between early axons are

273 classified as early. Contacts between middle axons or between early and middle axons are

274 classified as middle. Contacts between late axons, between early and late axons and between

275 middle and late axons are classified as late. Fraction of conserved early contacts is the number of

276 degree 4 early contacts divided by the total number of early contacts. Fraction of middle and late

277 conserved contacts are defined similarly.

\section{Data Availability}

279 The datasets generated and analyzed during the current study are available from the 280 corresponding author upon request. 


\section{References}

[1] Zheng Z, et al. (2018) A Complete Electron Microscopy Volume of the Brain of Adult Drosophila melanogaster. Cell 174(3):730-743.e22.

[2] Swanson LW, Lichtman JW (2016) From Cajal to Connectome and Beyond. Annual Review of Neuroscience 39(1):197-216.

[3] Sanes JR, Yamagata M (2009) Many Paths to Synaptic Specificity. Annual Review of Cell and Developmental Biology 25(1):161-195.

[4] Chedotal A, Richards $\sqcup$ (2010) Wiring the brain: The biology of neuronal guidance. Cold Spring Harbor Perspectives in Biology 2(6):a001917.

[5] White JG, Southgate E, Thomson JN, Brenner S (1986) The Structure of the Nervous System of the Nematode Caenorhabditis elegans. Philosophical Transactions of the Royal Society B Biological Sciences 314(1165):1-340.

[6] Cook SJ, et al. (2019) Whole-animal connectomes of both Caenorhabditis elegans sexes. Nature 571(7763):63-71.

[7] Brittin CA, Cook SJ, Hall DH, Emmons SW, Cohen N (2020) Beyond the connectome: A map of a brain architecture derived from whole-brain brain reconstructions. Under Review.

[8] Moyle MW, et al. (2020) Structural and developmental principles of neuropil assembly in C. elegans. bioRxiv p.2020.03.15.992222.

[9] Seiradake E, Jones EY, Klein R (2016) Structural perspectives on axon guidance. Annual Review of Cell and Developmental Biology 32:577-608.

[10] Sanes JR, Zipursky SL (2020) Synaptic specificity, recognition molecules, and assembly of neural circuits. Cell 181(3):536-56.

[11] Goodhill GJ (2016) Can Molecular Gradients Wire the Brain? Trends in Neuroscience 39(4):202-11.

[12] Aberle $\mathrm{H}$ (2019) Axon guidance and collective cell migration by substrate-derived attractants. Frontiers in Molecular Neuroscience 12:148.

[13] Honig B, Shapiro L (2020) Adhesion protein structure, molecular affinities, and principles of cell-cell recognition. Cell 181(3):520-35.

[14] Sanes JR, Zipursky SL (2010) Design Principles of Insect and Vertebrate Visual Systems. Neuron 66(1):15-36.

[15] Takemura S, et al. (2015) Synaptic circuits and their variations within different columns in the visual system of Drosophila. Proceedings of the National Academy of Sciences of the United States of America 112(44):13711-6. 
[16] Hedgecock EM, Culotti JG, Hall DH (1990) The unc-5, unc-6, and unc-40 genes guide circumferential migrations of pioneer axons and mesodermal cells on the epidermis in $C$. elegans. Neuron 4(1):61-85.

[17] Rapti G, Li C, Shan A, Lu Y, Shaham S (2017) Glia initiate brain assembly through noncanonical Chimaerin-Furin axon guidance in C. elegans. Nature Neuroscience 20(10):1350-1360.

[18] Kennerdell JR, Fetter RD, Bargmann CI (2009) Wnt-Ror signaling to SIA and SIB neurons directs anterior axon guidance and nerve ring placement in $C$. elegans. Development 136(22):3801-3810.

[19] Blankenship JT, Backovic ST, Sanny JS, Weitz O, Zallen JA (2006) Multicellular Rosette Formation Links Planar Cell Polarity to Tissue Morphogenesis. Developmental Cell 11(4):459-470.

[20] Harding MJ, McGraw HF, Nechiporuk A (2014) The roles and regulation of multicellular rosette structures during morphogenesis. Development 141(13):2549-58

[21] Williams M, Yen W, Lu X, Sutherland A (2014) Distinct apical and basolateral mechanisms drive planar cell polarity dependent convergent extension of the mouse neural plate. Developmental Cell 29(1):34-46.

[22] Shah PK, et al. (2017) PCP and SAX-3/Robo Pathways Cooperate to Regulate Convergent Extension-Based Nerve Cord Assembly in C. elegans. Developmental Cell 41(2):195-203.e3.

[23] Fan L, Kovacevic I, Heiman MG, Bao Z (2019) A multicellular rosette-mediated collective dendrite extension. eLife 8 pii: e38065. doi: 10.7554/eLife.38065.

[24] Shah PK, et al. (2017) An In Toto Approach to Dissecting Cellular Interactions in Complex Tissues. Developmental Cell 43(4):530-540.e4.

[25] Pare AC, et al. (2014) A positional Toll receptor code directs convergent extension in Drosophila. Nature 515(7528):523-527.

[26] Altun Z, Hall D (2012) WormAtlas.

[27] Santella A, et al. (2015) WormGUIDES: An interactive single cell developmental atlas and tool for collaborative multidimensional data exploration. BMC Bioinformatics 16: 189. doi: 10.1186/s12859-015-0627-8.

[28] Zallen JA, Yi B, Bargmann Cl (1998) The Conserved Immunoglobulin Superfamily Member SAX-3/Robo Directs Multiple Aspects of Axon Guidance in C. elegans. Cell 92(2):217-227. 
[29] Wang J, Ding M (2018) Robo and Ror function in a common receptor complex to regulate Wnt-mediated neurite outgrowth in Caenorhabditis elegans. Proceedings of the National Academy of Sciences of the United States of America 115(10):E2254-E2263.

[30] Gray JM, Hill JJ, Bargmann Cl (2005) A circuit for navigation in Caenorhabditis elegans. Proceedings of the National Academy of Sciences of the United States of America 102(9):3184-91.

[31] Williams BD, Waterston RH (1994) Genes critical for muscle development and function in Caenorhabditis elegans identified through lethal mutations. Journal of Cell Biology 124(4):475-490.

[32] White J, Southgate E, Thomson J, Brenner S (1983) Factors That Determine Connectivity in the Nervous System of Caenorhabditis elegans. Cold Spring Harbor Symposia on Quantitative Biology 48:633-640.

[33] Kolotuev I (2014) Positional correlative anatomy of invertebrate model organisms increases efficiency of TEM data production. Microscopy and Microanalysis 20(5):13921403.

[34] Burel A, et al. (2018) A targeted 3D EM and correlative microscopy method using SEM array tomography. Development 145(12) pii: dev160879. doi: 10.1242/dev.160879. [35] Cardona A, et al. (2012) TrakEM2 Software for Neural Circuit Reconstruction. PLoS ONE 7(6):e38011. 


\section{Acknowledgments}

367 We thank Netta Cohen, Scott Emmons, David Hall and Steven Cook for sharing their results on

368 the structural and functional analysis of the nerve ring and the connectome, members of the

369 UNIL EM facility for technical help, and the Caenorhabditis Genetic Center (funded by NIH

370 Office of Research Infrastructure Programs P40 OD010440) for C. elegans strains. Research in

371 the Z.B, D.A.C-R. and W.A.M. labs were supported by NIH grant No. R24-OD016474, and H.S. lab

372 by the intramural research program of NIBIB, NIH. Research in Z.B. lab was further supported by

373 an NIH center grant to MSKCC (P30CA008748). Research in the D.A.C.-R. lab was further

374 supported by NIH R01NS076558, DP1NS111778 and by an HHMI Scholar Award. H.S. and

375 D.A.C-R. acknowledge the Whitman and Fellows program at MBL. A.S. was supported by grant

376 2019-198110 (5022) from the Chan Zuckerberg Initiative and the Silicon Valley Community

377 Foundation. M.W.M was supported by NIH F32-NS098616.

\section{Author Contributions}

379 Designed experiments: C.B., A.S, K.B., I.K., W.A.M., H.S., D.A.C-R., Z.B. Performed biological and 380 imaging experiments: C.B., K.B., M.W.M., L.F., I.K. Performed image and data analysis: C.B., A.S., 381 K.B., M.W.M., L.F., R.C., I.K. Prepared manuscript: C.B., A.S., K.B., Z.B. (with assistance from all 382 authors). Supervised research: I.K., W.A.M., H.S., D.A.C-R., Z.B. Directed research: Z.B.

\section{Competing Financial Interests}

384 The authors declare no competing financial interests. Correspondence and requests for 385 materials should be addressed to Z.B. (baoz@mskcc.org). 
bioRxiv preprint doi: https://doi.org/10.1101/2020.05.27.119750; this version posted May 28, 2020. The copyright holder for this preprint (which was not certified by peer review) is the author/funder. All rights reserved. No reuse allowed without permission.
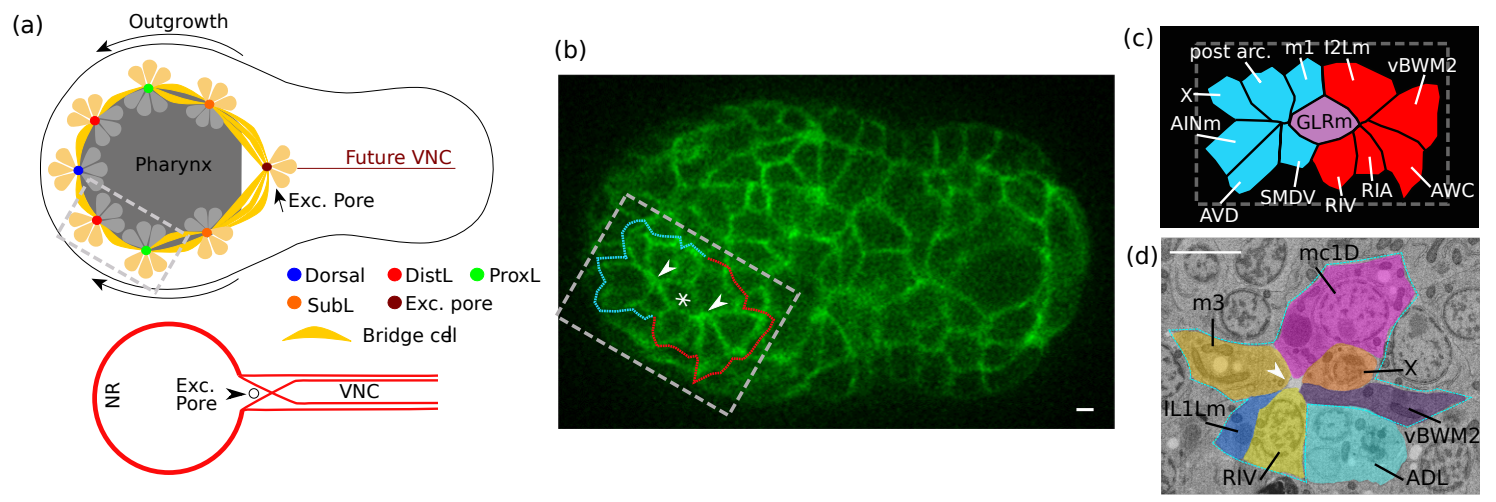

Figure 1. Nerve ring neurons form a ring of rosettes prior to axon outgrowth. (a) Top: schematic showing the spatial arrangement of 8 multicellular rosettes along the future nerve ring trajectory prior to innervation. Thin black line represents embryo contour. Ventral view, anterior to left. Color dots represent the center of each rosette. Thick arrows indicate the major direction of axon outgrowth. The DistL and ProxL rosettes in the grey rectangle are further shown in (b) and (c). For fluorescence images and cell composition of each rosette, see Fig S1 and Table S1. Bottom: schematic showing the final central nervous system. The posterior side of the excretory pore is where axons exit the nerve ring to enter the ventral nerve cord (VNC). (b) Fluorescence image of an embryo ( $350 \mathrm{mpf}$ ) with cell membrane label. Ventral view, anterior to left. Grey box corresponds to that in (a). Dashed line and arrowheads mark the contour and center of DistL (cyan) and ProxL (red) rosettes, respectively. * marks a bridge cell linking the DistL and ProxL rosettes. Scale bar: $1 \mu \mathrm{m}$. (c) Annotation for the region in the grey box in (b). Cyan marks DistL cells, red ProxL, and purple the bridge cell. Cell identities were determined through live imaging based lineage tracing. X marks the cell ABaraaaapaa, sister of e1D and eventually dies. (d) An EM image of the ProxL rosette. Dashed line and arrowhead mark the contour and center, respectively. Cell identities were determined by comparing cell positions to those in the WormGUIDES app [27] and considering known cell morphologies. X marks the cell ABalpaaapap, which eventually dies. Not all cells in the ProxL rosette are visible in this plane. The EM image sections the rosette from a different angle than (b) and hence shows different cells. Scale bar: $1 \mu \mathrm{m}$. 
(a)

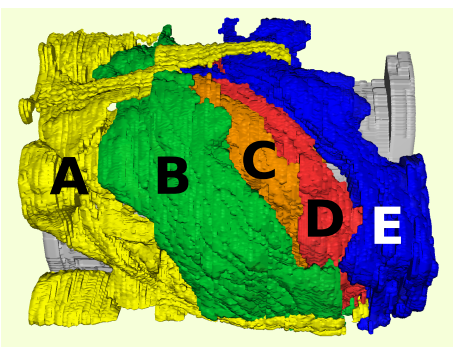

(b)

\begin{tabular}{|l|l|l|l|l|}
\hline & Dorsal & DistL & ProxL & SubL \\
\hline Stratum A & RMED, RME & & & \\
\hline Stratum C & & & & $\begin{array}{l}\text { SIADN, } \\
\text { SIBDN }\end{array}$ \\
\hline Stratum B & & & $\begin{array}{l}\text { RIV, SMDV, } \\
\text { SAAV }\end{array}$ & SMDD, AVK \\
\hline Stratum D & & AVD, AVJ & & \\
\hline Stratum E & ALA & & & \\
\hline
\end{tabular}

Motoneuron, Interneuron (c)

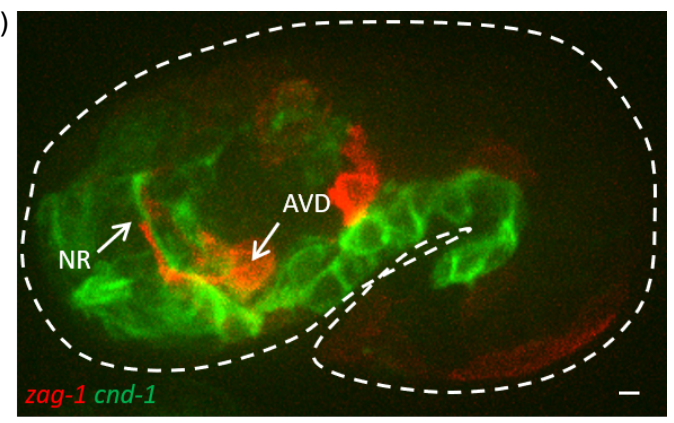

(d)

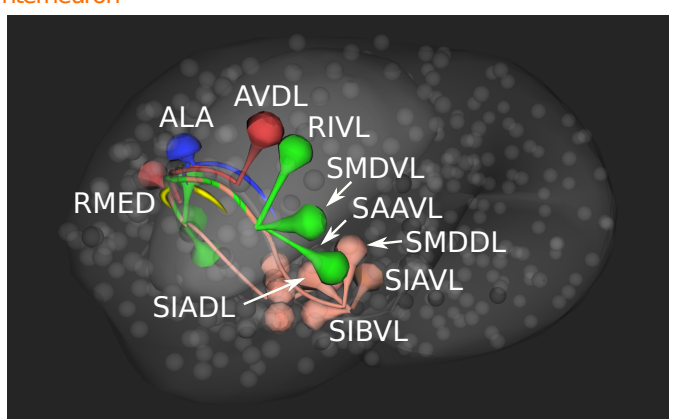

(e)

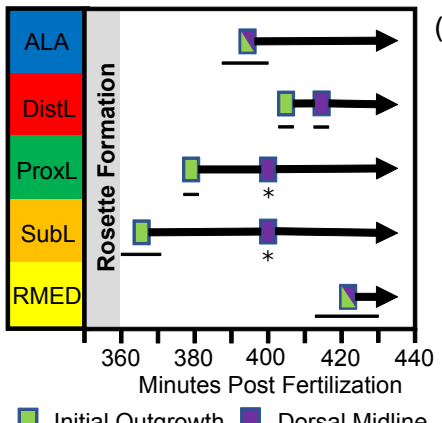

(f)

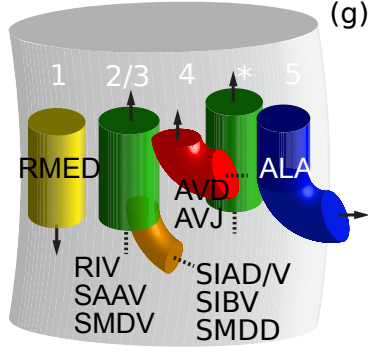

(g)

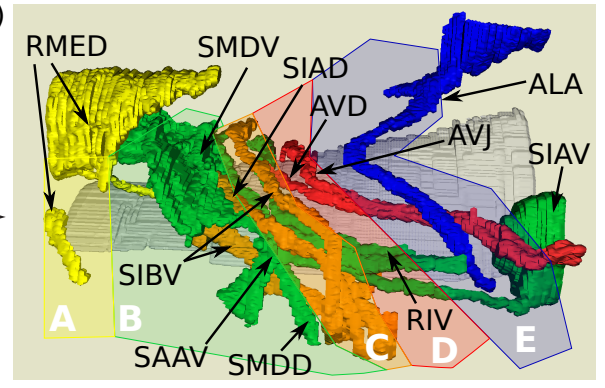

Figure 2. Rosette axons form the topographic scaffold of the nerve ring. (a) Rendering of the strata in the nerve ring from serial section EM of an L4. A-E label the individual stratum. B and C, which contain head motoneurons and sublateral motoneurons along with their closely associated interneurons, respectively, may be merged into one stratum depending on clustering threshold and weighting of axon contact [7,8]. (b) Correspondence between rosettes and nerve ring/connectome structures. (c) Fluorescence image of a 1.5-fold stage embryo (450 mpf). Left view, anterior to the left. Dashed white line shows the contour of the embryo. Note that zag-1p::PH::GFP is artificially colored red and $c n d-1 p:: \mathrm{PH}:: \mathrm{mCherry}$ green to follow the convention in (d) and (f). Scale bar: $1 \mu \mathrm{m}$. (d) Rendering of the 1.5-fold stage embryo. Anterior left view. Generated from the WormGUIDES app [27]. Spheres (semi-transparent) represent nuclei, which are based on systematic cell tracking and lineaging in an individual embryo. Large white structure shows the contour of the pharynx. Teardrop shapes are used to denote soma of select neurons. Cell names are given for neurons on the left side. Thin threads model axon outgrowth, which were traced in different embryos with fluorescent markers, and aligned both temporally and spatially to the nuclei dataset. Neurons are colored by the rosettes. Soma of RMED (yellow) is omitted for visual clarity. ALA and RMED are on the dorsal midline. (e) Temporal dynamics of axon outgrowth. Horizontal bars show the range of the observed timing $(n=3)$. The timing for the bilateral ProxL axons to meet at the dorsal midline (*) is used to align different embryos. (f) Schematic illustrating placement of early rosette axons in the nascent nerve ring, triangulated from two-color fluorescence imaging (Fig S2). Colors follow the strata in (a). Arrows indicate direction of axon growth. SubL axons (orange) converge to ProxL axons (green). Available cell-specific markers can not separate the two bundles after this point. * marks an unidentified axon bundle. (g) Rendering of rosette axons in the L4 nerve ring from serial section EM. Dorsal-left view. Axon colors follow the corresponding strata in (a). Grey is the pharynx. Semi-transparent blocks (labeled A-E) approximate the spatial ranges of the strata. See Fig S2 for rendering of the strata from the same perspective. RMED axon is not connected to the soma due to missing data. SIBV axon has two branches. 
(a)
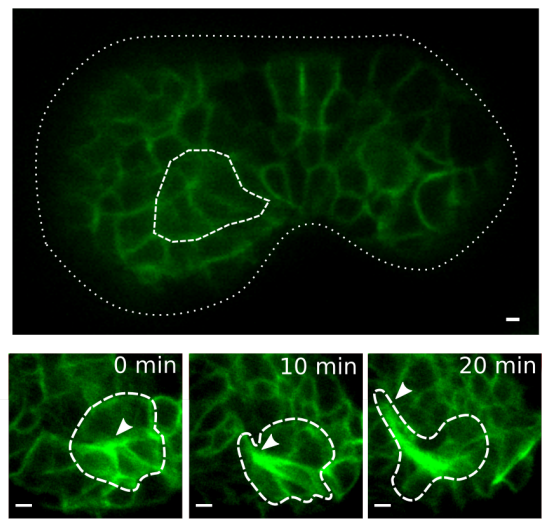

(b)

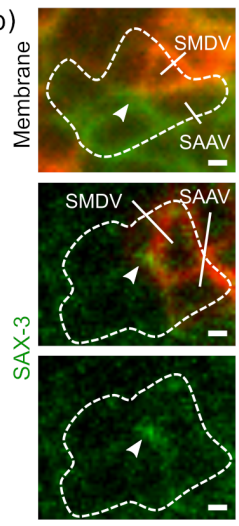

(c)

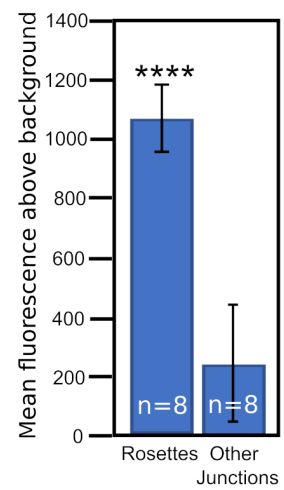

(d)

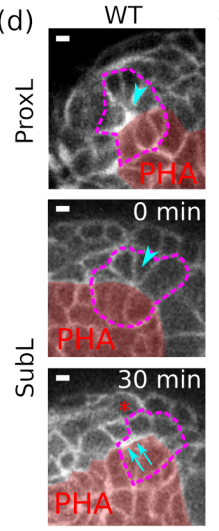

sax-3(ky123)

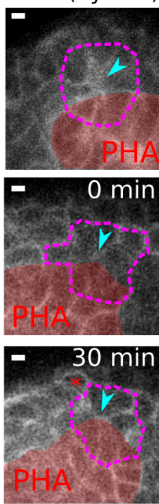

(e)

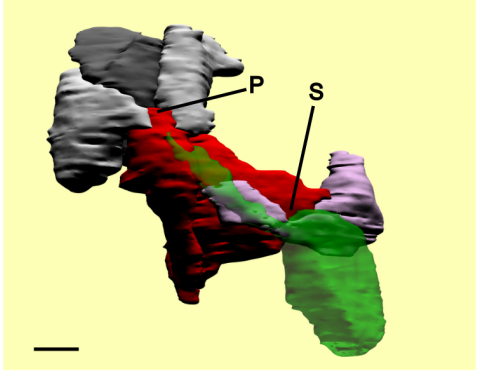

(f)
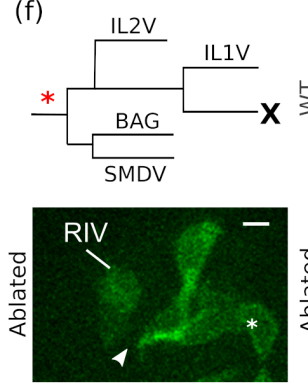
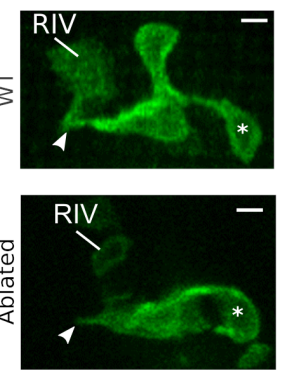

(g)

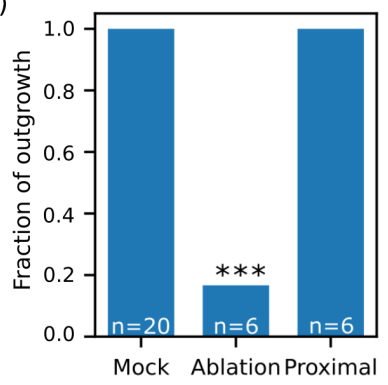

Figure 3. Organization of rosette axon outgrowth. (a) Axons grow from rosette center. Images of fluorescently labeled cell membrane showing outgrowth from the SubL rosette. Top: position of the SubL rosette (thick dashed line) in the embryo (thin dashed line for contour). Scale bar: $1 \mu \mathrm{m}$. Bottom: select time points, arrows point to rosette center $(0$ min $)$ and leading edge of growing axons (10 and 20 minutes). (b) SAX-3 localizes to rosette centers. Top: Membrane GFP shows the stereotypical position of the ProxL rosette (dashed line) next to a patch of cells (red) expressing cnd-1p::PH::mCherry. Cells of the ProxL rosette labeled by mCherry are named. Arrowhead points to rosette center. Middle: SAX-3::GFP localizes to the putative ProxL rosette center (arrowhead). Named cells were used as spatial landmark. Bottom: SAX-3::GFP channel alone. Scale bar: $1 \mu \mathrm{m}$. See Fig S3 for SAX-3 localization in other rosettes. (c) Quantification of SAX-3::GFP intensity at rosette centers and regular cell-cell junctions with 3 or 4 cells. Error bars represent 1 s.d. $p=4.7 \times 10^{-8}$ by $t$-test. See Methods for details. (d) Phenotypes in sax-3(ky123) mutants. Membrane GFP images. Top: The ProxL rosette (purple dash) has a less focused center (arrowhead) and was displaced from the boundary of the pharynx (PHA, red patch) as compared to the wild type (WT). Bottom: The posterior precursor SubL rosette forms as in the WT ( 0 min, arrowheads point to rosette centers). In the WT, it converges into SubL and generates axons (30 min, arrows point to thickened membrane signal of axons along the pharyngeal boundary), but in mutant the rosette remains an isolated center (30 min, arrowhead). Red * indicates the position of the amphid as a spatial landmark. Scale bar: $1 \mu \mathrm{m}$. (e) Volumetric reconstruction from serial sectioned EMs shows initial SIAD (green) and SIBD (purple) outgrowth from the SubL rosettes (s, grey cells on the right) along the bridge cells (IL1Vm and IL2, red) towards the ProxL rosette (p, grey cells on left). Scale bar: $1 \mu \mathrm{m}$. (f) Ablation of bridge cell precursor leads to failure of SubL outgrowth. Upper left: target cell of laser ablation $\left(^{*}\right)$ and its sublineage. Other: Images of SubL and ProxL neurons based on lim-4p::PH::GFP. Arrowheads point to axon tips from SubL neurons. * labels SIAV. In WT, the outgrowth converges with RIV from the ProxL rosette. In ablated embryos, SubL outgrowth is short and fails to reach the ProxL rosette. Image of the second ablated embryo (bottom right) was rotated 45 degrees along the anterioposterior axis for visual clarity. Ablations were performed on one side of the embryo, while the contralateral side was used as an internal negative control. Scale bar: $1 \mu \mathrm{m}$. (g) Fraction of proper SubL outgrowth under different conditions. Mock: no ablation. Proximal: a cell adjacent to the intended target in (f) was ablated, to control for potential off-target effects. $* * *$ denotes statistically different from mock, $p=5.8 \times 10^{-8}$ by $t$-test, assuming a binomial distribution (i.e. axons either do or do not exhibit proper outgrowth). 


\section{(a)}

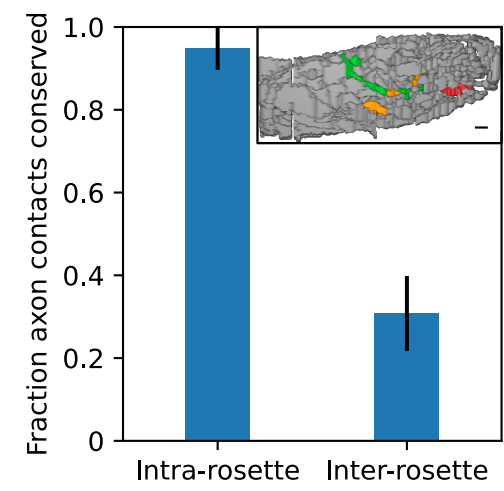

(b)

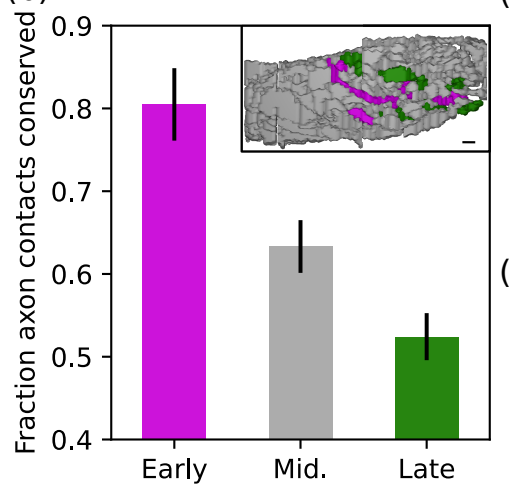

(c)

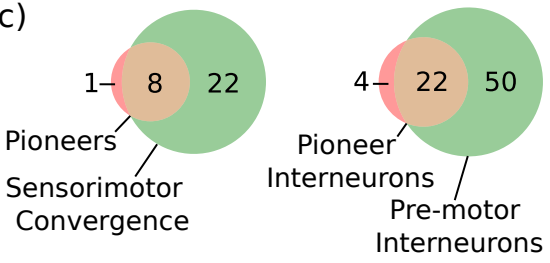

(d)

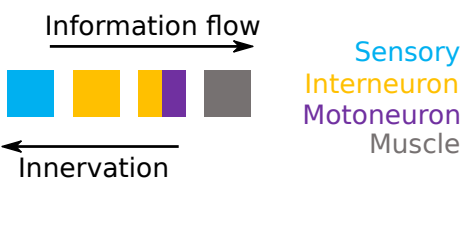

Figure 4. Rosettes reveal additional organizational schemes. (a) Fraction of conserved axon contact sites within $(n=19$ contacts) and between ( $n=26$ contacts) rosettes based on EM from the larval nerve ring. Inset: oblique slice of the volumetric rendering of the nerve ring (Fig 2a), taken $3.5 \mu \mathrm{m}$ to the right of the dorsal midline. Coloring of rosette axons is identical to Fig 2a, with non-rosette axons colored gray. (b) Fraction of intra-stratum axon contact sites based on time of nerve ring innervation for early ( $n=88$ contacts, purple), middle ( $n=229$ contacts, gray) and late ( $n=309$ contacts, green) axons. For definition of the early, middle and late groups of axons, see text. Inset: same oblique slice as in (a), but with axons color based on time of innervation. $(\mathrm{a}, \mathrm{b})$ Error bars denote the standard error, which were computed assuming a binomial distribution (i.e. contacts are either conserved or not). Inset scale bar: $1 \mu \mathrm{m}$. (c) Left: Fraction of rosette interneurons that correspond to the sensory convergence layer in ResNet analysis [7]. Right: Fraction of rosette interneurons that correspond to the IN1 and IN2 pre-motor interneuron layers defined in [6]. (d) A schematic for functional layers in the connectome and growth pattern of the nerve ring. 